\title{
Bone Mass Distribution of the Distal Tibia in Normal, Osteopenic, and Osteoporotic Conditions: An Ex Vivo Assessment Using HR-pQCT, DXA, and Computational Modelling
}

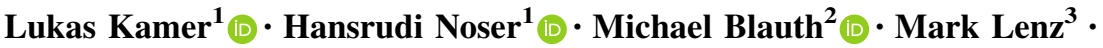 \\ Markus Windolf ${ }^{1}$ (D) $\cdot$ Albrecht W. Popp $^{4}$ (I)
}

Received: 10 March 2016/Accepted: 8 August 2016/Published online: 30 August 2016

(c) Springer Science+Business Media New York 2016

\begin{abstract}
Osteoporosis leads to bone loss and structural deterioration, which increase the risk of fractures. The aim of this study was to characterize the three-dimensional (3D) bone mass distributions of the distal tibia in normal, osteopenic, and osteoporotic conditions. High-resolution peripheral quantitative computed tomography (HR-pQCT) of the $33 \%$ of the distal tibia and local dual-energy X-ray absorptiometry were applied to 53 intact, fresh-frozen tibiae. The HR-pQCTs were graded to assign local $T$-scores and merged into three equally sized average normal, osteopenic, and osteoporotic surface models. Volumetric bone mineral density (vBMD) was determined using categorized $T$-scores, volumetric visualization, and virtual bore probes at the dia-, meta-, and epiphyseal sites (T-DIA, T-META, and T-EPI). We observed a distinct 3D bone mass distribution that was gradually uninfluenced by $T$ score categories. T-DIA was characterized by the lowest bone mass located in the medullary cavity and a wide homogenous cortex containing the maximum vBMD. The T-META showed decreased cortical thickness and maximal vBMD. At the T-EPI, the relatively low vBMD of the mostly trabecular bone was similar to the maximal cortical vBMD in this sub-region. Four trabecular regions of low
\end{abstract}

Lukas Kamer

lukas.kamer@aofoundation.org

1 AO Research Institute Davos, Clavadelerstrasse 8, 7270 Davos, Switzerland

2 Department of Trauma Surgery and Sports Medicine, Medical University of Innsbruck, 6020 Innsbruck, Austria

3 Department of Trauma, Hand and Reconstructive Surgery, University Hospital of Jena, 07747 Jena, Germany

4 Department of Osteoporosis, Inselspital, Bern University Hospital, University of Bern, 3010 Bern, Switzerland bone mass were identified in the recesses. The bone content gradually decreased at all sites, whereas the pattern of bone mass distribution remained essentially unchanged, with the exception of disproportionate losses at T-DIA, T-META, and T-EPI that consistently showed increased endocortical, intracortical, and trabecular bone loss. Extra information can be obtained from the specific pattern of bone mass distribution, potential disproportionate bone losses, and method used.

Keywords Osteoporosis - Distal tibia - Bone mineral density $\cdot$ DXA $\cdot$ HR-pQCT

\section{Introduction}

Osteoporosis leads to bone loss and structural decay [1] and exerts a variety of other effects on the skeletal system. Deleterious effects, such as increased endocortical resorption, increased cortical porosity, and greatly decreased bone mineral density (BMD), cause fragility fractures in elderly patients. These effects may be partially compensated for by periosteal apposition with outward cortical displacement [2]. Furthermore, skeletal site-specific variations may exist, but the current data remain unclear.

High-resolution peripheral quantitative computed tomography (HR-pQCT) is a reliable imaging modality that is widely used for the volumetric quantification of bone structures and properties and has a voxel resolution of $82 \mu \mathrm{m}$ [3-6]. It provides detailed spatial information of the bone as volumetric BMD data. Standard clinical HR-pQCT protocols include the scanning and analysis of a given region of interest (ROI) $(9.02 \mathrm{~mm}$ in the $z$-axis beginning $22.5 \mathrm{~mm}$ proximal to the distal tibial endplate), but do not describe the entire skeletal site. However, the World Health 
Organization uses a densitometric definition of osteoporosis based on areal BMD (aBMD) measured with dual-energy X-ray absorptiometry (DXA) [7], which remains the gold standard for diagnosing osteoporosis.

In the appendicular skeleton, the metaphyseal sites of long bones are predominantly affected by fragility fractures. Even though fragility fractures of the distal tibia are uncommon, they are particularly challenging injuries to treat, and the number of such injuries is expected to increase in proportion to the ageing of the world population [8]. The distal tibia is an interesting site to study because it is characterized by a substantial amount of cortical and trabecular bone and its straight shape allows aBMD to be assessed without being influenced by the exterior morphology [9]. In addition, local aBMD values have been reported [10], and the entire region and intact bone specimens can be scanned using a custom HR-pQCT scanner [11]. The distal tibia is a weight-bearing extremity that supports a large portion of the person's body weight. Interestingly, because the epi- and metaphyses contain primarily trabecular bone, they do not simply collapse as an osteoporotic vertebra does, and fractures at the distal tibia occur at the epi- or metaphyseal sites [12]. One cohort study of postmenopausal women demonstrated the predictive value of low aBMD at the distal tibia for evaluating the potential risk of fracture [13].

The amount of bone loss is highly variable between different individuals, and certain skeletal sites may exhibit different bone mass distributions and bone loss patterns. This makes demonstrating the characteristics of bone mass distribution and bone loss for an entire skeletal site challenging.

Detailed characterizations of the three-dimensional (3D) bone structure for this skeletal site and the changes that occur during bone loss have not yet been reported. Substantial differences in bone mass at different skeletal sites and within sub-regions (i.e. at diaphyseal, metaphyseal, and epiphyseal levels) may be expected. However, the differences still need to be further analysed in normal, osteopenic, and osteoporotic conditions. In addition to an in vivo study performed by Boutroy et al. [3] on the trabecular bone microarchitecture, calculating the 3D anatomy of the entire distal tibia length and demonstrating the distribution of its 3D bone mass in normal, osteopenic, and osteoporotic conditions may be a useful approach for describing the anatomical variations at these sites and how they are associated with the risk of fracture allowing the treatment of fragility fractures to be improved in the future.

The objective of the present study was to characterize the $3 \mathrm{D}$ anatomy of the distal tibia and to map the bone mass of the distal tibia in normal, osteopenic, and osteoporotic conditions.

\section{Materials and Methods}

\section{Bone Specimens}

Fifty-three intact, fresh-frozen post-mortem tibia specimens were analysed. The bones were unpaired specimens (47 left and six right) from 26 female and 27 male donors aged 28-96 years (mean \pm SD combined population $75 \pm 12.5$ years; females $78.9 \pm 9.2$ years; and males $71.3 \pm 14.2$ years). The bone specimens were obtained defleshed and then frozen for unrelated biomechanical studies after internal approval by the Scientific Review Board of the AO Research Institute Davos and were made available for scanning for the purpose of this project. An ethical waiver was provided by the local ethical committee stating that this study did not require an ethical approval. Donors who died from illnesses other than bone diseases were included in this study. Conventional plain $\mathrm{X}$-rays were taken of all the tibiae prior to processing to exclude tibiae showing alterations that were not part of the patient's history, such as unidentified bone disease in the regions of interests. The specimens were stored at $-20{ }^{\circ} \mathrm{C}$ until use. All of the samples were kept vacuum-packed in polyethylene during the measurement.

\section{HR-pQCT Scanning}

After thawing the specimens to room temperature, the distal $16 \mathrm{~cm}$ of the tibiae was scanned using HR-pQCT (XtremeCT $^{\mathrm{TM}}$, Scanco Medical, Brüttisellen, Switzerland) with the X-ray tube set at a peak voltage of 60 $\mathrm{kVp}$ and $900 \mu \mathrm{A}$ and an image matrix size of $1024 \times 1024$ at a nominal $82-\mu \mathrm{m}$ isotropic voxel resolution. A Scanco phantom standard was scanned daily for quality control. The manufacturer's software was used to generate the raw image data in Hounsfield unit (HU). The data were stored in Digital Imaging and Communications in Medicine (DICOM) format, transferred to a standard desktop computer, and loaded into Amira software $\left(\right.$ Amira $^{\circledR}$ version 5.4.3, Visualization Science Group, Merignac Cedex, France). Amira is a commercial available and extendable software for scientific visualization, for data analysis, and for the presentation of medical imaging data. The software can be extended by customized scripts and programming modules, if required. Negative $\mathrm{HU}$ values were not assigned to the bone tissue and were instead replaced with 0 . The volumetric BMD (vBMD) values are expressed as $\mathrm{mg}$ hydroxyapatite $(\mathrm{HA}) / \mathrm{cm}^{3}$ based on a linear transformation defined by the machine calibration. 


\section{DXA Measurements}

aBMD was measured by DXA at the distal tibia. The scans were performed by placing the vacuum-packed tibia specimens in a basin filled with semolina to simulate the soft tissue and scanned with a standard DXA device (Hologic QDR $4500 \mathrm{~A}^{\mathrm{TM}}$, Hologic, Bedford, MA, USA). The spine phantom was also scanned daily for quality control. The manufacturer's software was used to define the ROI using a standardized procedure, as previously described [14]. The aBMD values are expressed in $\mathrm{g} / \mathrm{cm}^{2}$. The $T$ scores were calculated as the number of standard deviations from the mean peak BMD of a reference population. A population of 400 Caucasian women and men, 20-80 years of age, living in the area of Berne, served as a reference [9]. Each specimen was evaluated twice by DXA, and the average values were used.

\section{Resampling and Grouping of the HR-pQCT Scans by $T$-Scores}

The HR-pQCT scans were resampled to a $0.164-\mathrm{mm}$ isotropic image resolution and grouped according to the $T$ score into normal, osteopenic, and osteoporotic categories. The group with normal $T$-scores consisted of 13 scans with a mean $\pm \mathrm{SD} T$-score of $-0.16 \pm 0.49$. $T$-scores indicating osteopenia were obtained from 25 scans $(-1.65 \pm 0.41)$, and scores indicating osteoporosis were observed for 15 samples $(-3.24 \pm 0.46)$.

\section{Average vBMD Values for the Normal, Osteopenic, and Osteoporotic Models}

All of the HR-pQCT scans were distally cropped to standardize images measuring $33 \%$ of the full tibial length. Image data for the right tibiae were mirrored to produce 'left' sides. Standard threshold-based image segmentation was performed to create 3D computer models of the bone surfaces. Geomagic software (Geomagic Studio version 12, Geomagic U.S. Corp., Research Triangle Park, NC, USA) was used to smooth and process the surfaces to obtain regular surfaces without holes or triangle intersections. In Amira software, homologous anatomical surface points and non-homologous segment point landmarks were manually placed (Fig. 1). The interpolated equidistant homologous segment points were then computed, and a representative sample was selected as a reference. This sample was processed to exhibit a specified number of surface points and triangles. Based on the reference, a thin plate spline (TPS) transformation [15] was applied to create homologous surface points with identical numberings and locations on the different models consisting of 5117 surface points and
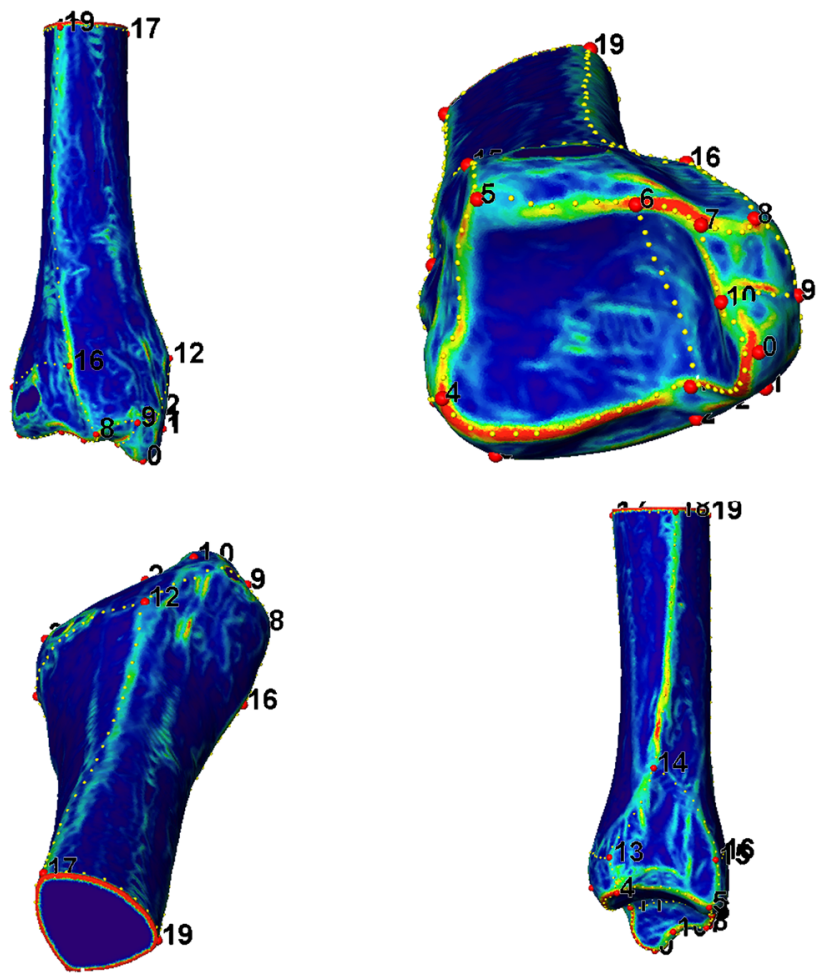

Fig. 1 Curvature-graded colourmap models of the left distal tibia showing the identification of anatomical landmarks and segments. A set of distinct anatomical homologous landmarks (red dots) were manually placed and connected by non-homologous segments points (yellow dots). From the latter, equidistant homologous points were computed that divided the distal tibiae into segments with identically numbered and located anatomical and segment points

10,190 triangles per distal tibia [16]. The homologous surface points were aligned using an unscaled generalized Procrustes fit alignment and averaged to generate a left mean surface model of the distal tibia [17].

We computed a regularly spaced grid placed within the mean surface model of the distal tibia with an isotropic voxel edge length of $0.164 \mathrm{~mm}$. This reference grid was warped to match the samples by TPS transformation based on the homologous surface points to obtain a homologous voxel grid structure for all samples. Accordingly, the volumetric data from all of the scans were reprocessed to obtain identically numbered and located anatomically homologous grid data. All of the data were elastically transformed onto the mean surface model. This procedure resulted in a volumetric dataset with $16,283,472$ averaged voxels located within the mean surface model of the distal tibia. To obtain averaged vBMD models of the normal, osteopenic, and osteoporotic HR-pQCT scan groups, this algorithm was repeated with the data from each of the three groups. These processes resulted in three distinct models of identical size, shape, and volume that differed in bone mass as described by the vBMD values. 


\section{Analysis}

We mapped the vBMD data in 3D and displayed their variations separately for the normal, osteopenic, and osteoporotic models. Using a volume-rendering technique, different vBMD threshold ranges were selected, and the upper threshold was gradually increased while maintaining the lower threshold at zero. For proper 3D visualization, we inverted the sign of the vBMD values using a scale factor of -1 (e.g. a vBMD value of 150 became -150 ). This procedure prevented the trabecular bone from being masked by the dense cortical shell.

To quantify the differences between the three groups in the different sub-regions of the distal third of the distal tibia, we measured the vBMD values along a 4-mm-diameter virtual bore probe at three selected sites. We calculated the averaged vBMD, cortical thicknesses (Ct.Ths), and maximal vBMD values at the T-DIA, T-META, and T-EPI sites based on the position of the bore probes using imaging data with an isotropic resolution of $82 \mu \mathrm{m}$. The bore probes were evaluated at anatomically corresponding sites in the midline, in a medial to lateral orientation, and at 114,45 , and $22 \mathrm{~mm}$ from the caudal end (also see Fig. 2, right illustrations). The bore probe radius was $0.5 \mathrm{~mm}$. In the cortex, the maximal vBMD values were found medially and laterally, and these values corresponded to the two peak values of the bore probe. In the trabecular bone, the vBMD was evaluated for a specified sub-volume located in the middle of the bore probe that corresponded to $40 \%$ of the total bore probe volume. Ct.Th was manually measured by the same observer at the opposite cortices, as illustrated by Fig. 3. The measurements were performed in anatomically corresponding HR-pQCT cross sections as given by a reference line. The location and orientation of the HR-pQCT cross sections were determined via a computerized process. The average vBMD value for each entire distal tibia was calculated and considered to be a second bone quality measure similar to the $T$-score.

\section{Statistical Analysis}

Data are presented as mean \pm SD. Descriptive statistics were determined for the vBMD data, and correlation coefficients were calculated between the averaged vBMD values and the other variables. We examined the correlations of several variables with the averaged vBMD to test the hypothesis that the mechanism of bone loss varies according to bone microstructure. Statistical comparisons between the correlations for the dependent and overlapping groups were calculated using one-tailed Meng's tests [18] to compare two overlapping [one variable in common (mean vBMD)] and dependent (from the same sample) correlations. $P$ values $<0.05$ were considered statistically significant.
Fig. 2 Virtual bore probing given in vBMD, evaluated at anatomically corresponding sites in the midline, in a medial to lateral orientation, and at 114, 45, and $22 \mathrm{~mm}$ from the caudal end. Mean vBMD values at the T-DIA, T-META, T-EPI sites along the lateral medial axis of the virtual bore probe categorized by normal bone mineral density (black line), osteopenia (dark grey line), and osteoporosis (light grey line) are shown. The data demonstrate mainly parallel curve patterns. Differences in the cortical peak vBMD values, cortical thicknesses, and trabecular vBMD values at the three levels indicate endocortical, intracortical, and trabecular bone loss

\section{Results}

The mean length of the averaged tibial model was $122 \mathrm{~mm}$, corresponding to $33 \%$ of the tibial length. The average vBMD of the distal tibiae was $463 \pm 56 \mathrm{mg} \mathrm{HA} / \mathrm{cm}^{3}$ in the normal group, $364 \pm 53 \mathrm{mg} \mathrm{HA} / \mathrm{cm}^{3}$ in the osteopenic group, and $244 \pm 50 \mathrm{mg} \mathrm{HA} / \mathrm{cm}^{3}$ in the osteoporotic group. The statistical analyses revealed that the sample groups were normally distributed with equal variances. The differences between the samples are visualized as box plots in Fig. 4. Because the notches did not overlap, the medians were significantly different $(P<0.05)$.

\section{Mapping the vBMD via Volume Rendering}

The bone mass distributions of the normal, osteopenic, and osteoporotic models were simultaneously visualized in 3D using narrow, intermediate, and wide threshold settings (Fig. 5). When a low and narrow threshold (0 to -2.5 vBMD) was selected, sites exclusively in the osteoporotic model were labelled. The labelled sites matched the areas located near to or within the medullary cavity. Upon expanding the threshold to 0 to $-11 \mathrm{vBMD}$, labelling was observed in all three of the models. However, the extent of the labelling was unequal, proving that the bone contents were different among the three models. Further expansion of the threshold level led to an expansion of the labelled area first in the lateral, and then predominantly in the distal, orientation. At a threshold of 0 to $-52 \mathrm{vBMD}$, the labelling reached the epiphysis in the osteoporotic model, but not in the other two models. We later identified four epiphyseal recesses in the osteoporotic model that had the lowest bone mass. Among these recesses, the medial malleolus was the first posterior spot to be labelled in the osteoporotic model at a threshold of 0 to $-20 \mathrm{vBMD}$. Between these four spots of low bone mass and the medullary cavity, a higher bone mass connecting the cortical bone of the diaphysis with the subchondral bone was observed. Additional sites with increased local bone mass were identified at the epiphyseal plate and the subchondral bone. At a threshold of 0 to -190 vBMD, the epiphyseal areas in all three bone models were labelled. Although the four areas of low bone mass 

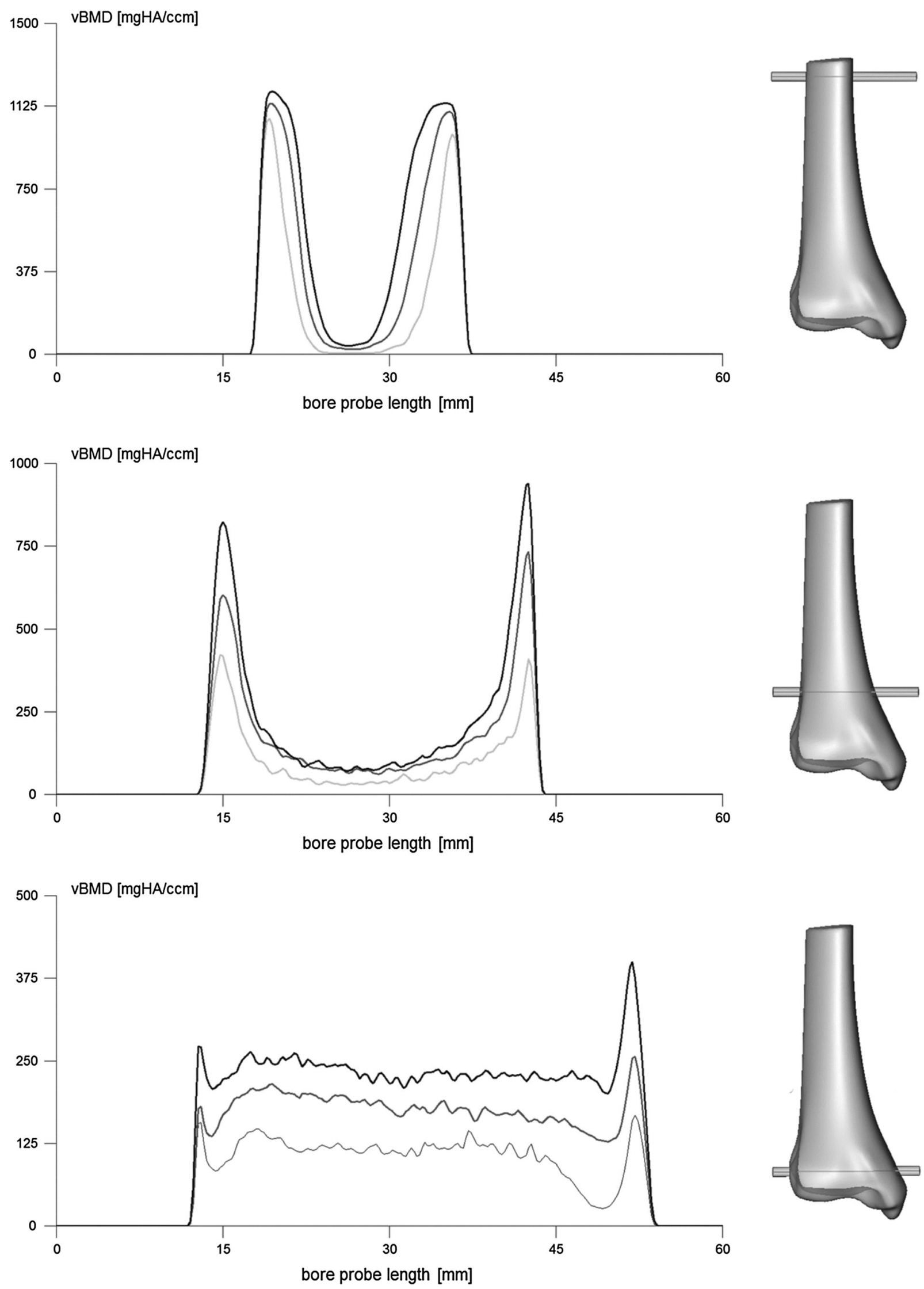


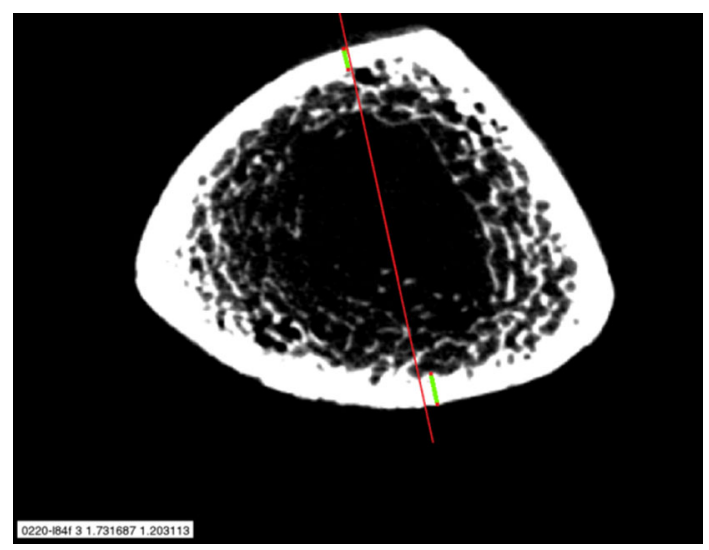

Fig. 3 Manual cortical thickness measurements (green lines with red dots) based on an axial HR-pQCT cross section at an anatomically corresponding region of the distal tibia. The labelling (below left) indicates the given cross section, and the red line illustrates the orientation of measurements within the cross section

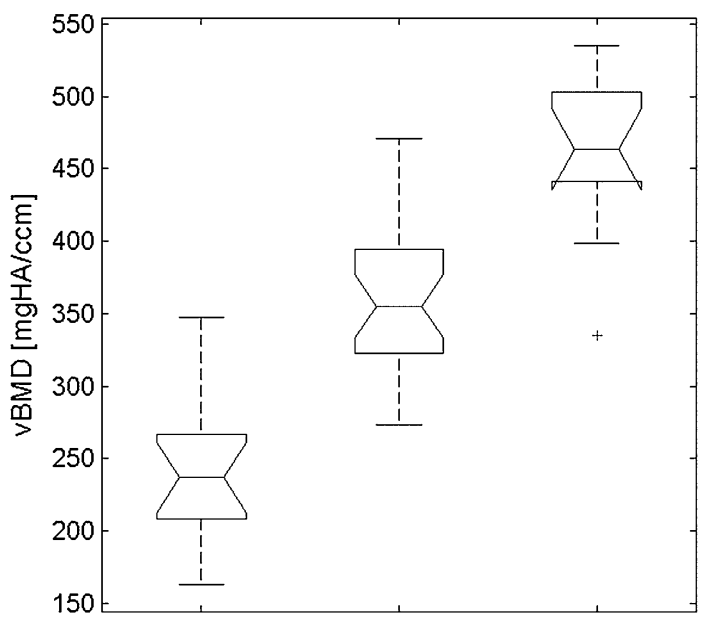

Fig. 4 Data overview with box plots of the average vBMD values of the distal tibiae in the osteoporotic (left), osteopenic (middle), and normal (right) categories given as median values (i.e. 242, 355, and $468 \mathrm{mgHA} / \mathrm{ccm}$ ). As the data are normally distributed, mean values (i.e. 248, 262, and $464 \mathrm{mgHA} / \mathrm{ccm}$ ) were also derived

located in the recesses remained disconnected in the normal and osteopenic models, they were fused in the osteoporotic model. At a vBMD threshold of approximately 0 to -300 vBMD, fusion was also observed in the normal model.

When a threshold of 0 to -500 vBMD was selected, only the cortical bone remained unlabelled (Fig. 6). This threshold clearly revealed differences in the thickness of the unlabelled cortical bone, which shrank from proximal to distal in all of the models. Furthermore, the cortical thickness decreased radially in the osteopenic and osteoporotic models compared to the normal model.

\section{Mapping the vBMD via Virtual Bore Probing}

The local bone masses available upon insertion of the virtual bore probe at the T-DIA, T-META, and T-EPI sites are given in vBMD, as illustrated in Fig. 2. Descriptive statistics of the averaged probe volumes are shown in Table 1 . All mean values at a given site were statistically different according to an unbalanced one-way analysis of variance (ANOVA) comparing two or more means of independent samples $(P<0.001)$. All sample populations were normally distributed (Lillifore's test, $P>0.09$ ) and had equal variance (Levene's test, $P>0.25$ ). Based on the statistics of the ANOVA, a multiple comparison test (MATLAB's multcompare function) was performed using the Bonferroni method. This method uses critical values from Student's $t$-distribution after an adjustment to compensate for multiple comparisons. All pairs of tested means were significantly different $(P<0.001)$. At the T-DIA site, there was virtually no bone mass available in the central bone. The maximal vBMD values were observed in the cortex, and no major differences in bone mass were found between the three groups at this location. In the normal model, however, the maximal vBMD value was found at a longer probe distance, which suggested a higher Ct.Th. At the T-META site, the maximal vBMD was substantially lower than that found at the T-DIA site, which also showed large differences among the three models. The medial bone had a higher vBMD than did the lateral bone, which is relieved by the fibula. At the T-EPI site, the virtual bore probes showed only a thin layer of the cortex and a low maximal vBMD. The vBMD values of the trabecular bone were unequally distributed throughout the probe lengths, and the values indicated variability within the trabecular bone.

To confirm that the observed differences in the mean vBMD values between the sub-regions were statistically significant, their correlations with Ct.Th, max vBMD, and mean vBMD in the middle of the bore probe were compared for each sub-region using Meng's tests [18]. At the primarily cortical bone-containing T-DIA site, the correlations between the average vBMD and the lateral (0.89) and medial (0.89) cortical thicknesses were significantly greater $(P<0.001)$ than those between the corresponding average vBMD and lateral (0.477) and medial (0.597) maximal vBMD.

At the T-META site, neither of the correlations mentioned above were significantly different. However, comparisons of the correlations between mean vBMD and maximal vBMD at T-DIA (0.849) and at T-META (0.597) revealed a significant difference at the medial site $(P<0.001)$ and a trend towards a difference laterally (0.587 vs. $0.477, P=0.174)$. 

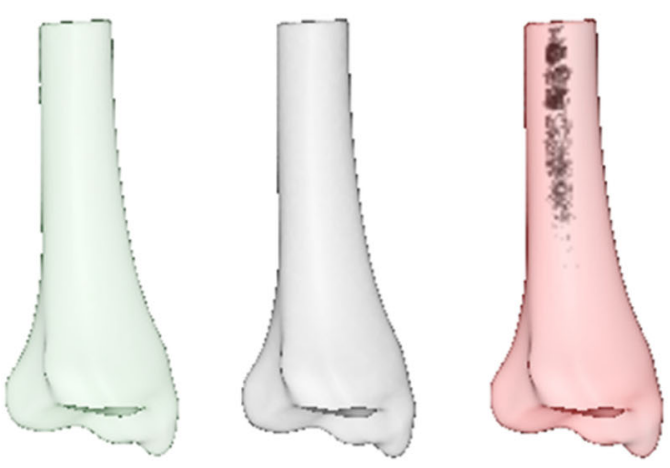

a
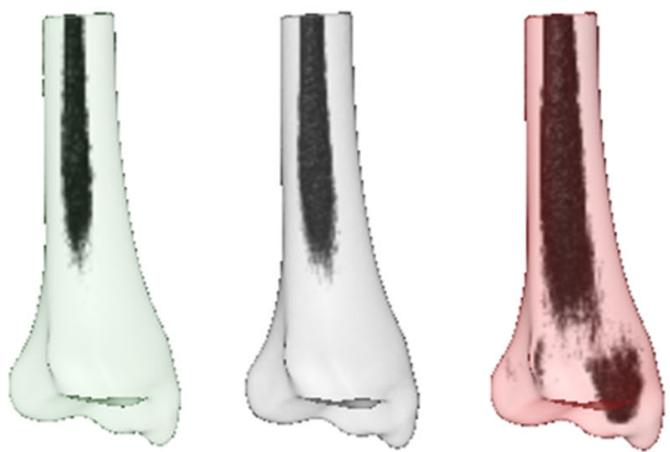

C

Fig. 5 Four thresholds were selected and visualized using volumerendering techniques to illustrate the different vBMD patterns in the averaged normal (green), osteopenic (grey), and osteoporotic (red)

Fig. 6 Visualization of the cortical thicknesses over a wide threshold ( 0 to -500 inverted vBMD) in the averaged normal (green), osteopenic (grey), and osteoporotic (red) bone models
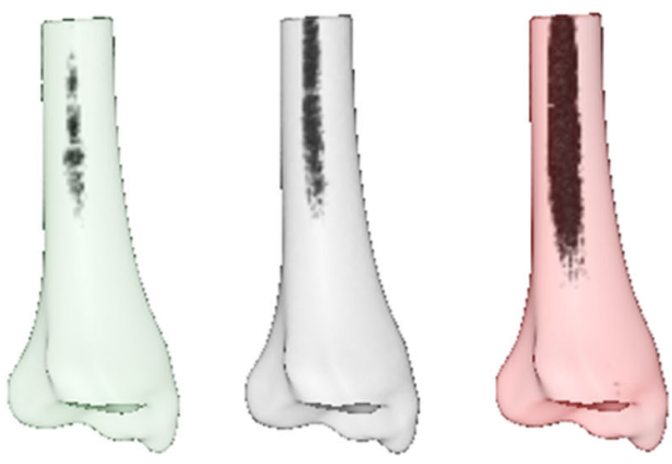

b
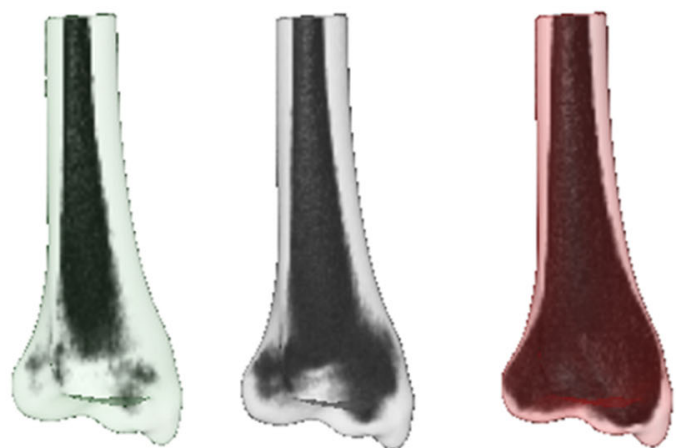

d

bone models a 0 to -2.5 inverted vBMD, $\mathbf{b} 0$ to -11 inverted vBMD, c 0 to -52 inverted vBMD, and $\mathbf{d} 0$ to -190 inverted vBMD

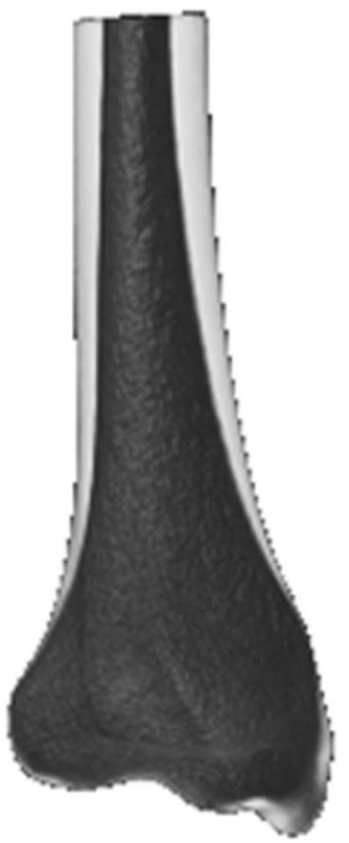


Table 1 Descriptive statistics of the virtual bore probes

\begin{tabular}{|c|c|c|c|c|}
\hline & Mean vBMD $\left(\mathrm{mg} \mathrm{HA} / \mathrm{cm}^{3}\right)$ & SD & Min & $\operatorname{Max}$ \\
\hline \multicolumn{5}{|l|}{$T-D I A$} \\
\hline Normal aBMD & $645(100 \%)$ & 98 & 415 & 779 \\
\hline Osteopenic aBMD & $493(76 \%)$ & 108 & 389 & 701 \\
\hline Osteoporotic aBMD & $313(48 \%)$ & 90 & 184 & 548 \\
\hline \multicolumn{5}{|l|}{$T-M E T A$} \\
\hline Normal aBMD & $259(100 \%)$ & 41 & 157 & 320 \\
\hline Osteopenic aBMD & $199(77 \%)$ & 28 & 141 & 256 \\
\hline Osteoporotic aBMD & $123(47 \%)$ & 31 & 82 & 206 \\
\hline \multicolumn{5}{|l|}{$T-E P I$} \\
\hline Normal aBMD & $244(100 \%)$ & 47 & 147 & 306 \\
\hline Osteopenic aBMD & $181(74 \%)$ & 34 & 104 & 239 \\
\hline Osteoporotic aBMD & $111(45 \%)$ & 31 & 75 & 200 \\
\hline
\end{tabular}

Values were assessed at three locations (T-DIA, T-META, T-EPI) and calculated for the three groups as sampled according to aBMD values. Values are shown with consistent percentages of vBMD compared with normal vBMD
At the T-EPI site, which contained the lowest absolute vBMD levels and minimal differences between the cortical and trabecular bone, no significant differences between the correlations between overall mean vBMD and mean vBMD in the centre of the T-EPI ( 0.643 vs. $0.624, P=0.427)$ or with the medial or lateral maximal vBMD (0.643 vs. 0.654 , $P=0.540)$ were observed.

\section{Discussion}

This anatomical study was performed to characterize the bone mass and distribution in the distal tibia in normal, osteopenic, and osteoporotic conditions.

We used aBMD and vBMD data and image processing techniques to assess the bone mass and bone loss in 3D, similar to the techniques used in a report by Kamer et al. [11]. In the present study, we combined evidence from HRpQCT and DXA imaging. We used post-mortem bone samples and extended the HR-pQCT protocol to image the distal third of the tibia. The data derived from these scans were grouped according to the $T$-scores and averaged to obtain normal, osteopenic, and osteoporotic bone models. This method involved computational techniques for creating bone models identical in shape, size, and volume, but with variable bone density. This allowed us to analyse corresponding anatomical sites, which is a key element of statistical modelling [19-21].

Determining BMD with DXA or HR-pQCT is an established and well-documented method. According to a study by Wähnert et al. [22] on the influence of bone temperature on the measured bone mineral density via a DXA evaluation, we performed all the measurements in a standardized and thawed condition.
We experienced computational and numerical difficulties while processing the large datasets we collected using a standard desktop computer. To solve this problem, we downsized the HR-pQCT data to an image resolution of $0.164 \mathrm{~mm}$ after setting the values for the tissues other than bone to 0 . Normally, areas of high density are less transparent than areas of low density. The volume-rendering method, as given by Amira software, is not suitable for the visualization of bony regions with low bone density because such regions are masked by the dense cortical shell. Therefore, we introduced a method for visualizing low bone mass areas by selecting a scale factor of -1 , thus inverting the vBMD values. Then, we used a texture-based volume rendering with specular shading effects and a transparency constant-colour colourmap. Consequently, the isosurface of the corresponding inner bone quality could be visualized with a variable lower limit of this colourmap without being masked by the high cortical values surrounding the interior bone parts. Of course, other techniques could be used to achieve similar rendering results. Varying the lower thresholds allowed us to identify and visualize the sites with low bone mass in 3D.

We identified a characteristic pattern in the trabecular and cortical bone mass distribution, which varied across the anatomical sites examined. In the presence of osteopenia and osteoporosis, the bone mass was ubiquitously decreased compared to the normal model, while the pattern of bone mass distribution remained constant. We found no sites with invariable bone mass. As expected, the loci with the lowest vBMD values were close to the medullary cavity.

In the epiphysis, the labelling process began at a higher vBMD and did not originate from a single central spot, but rather from four peripheral spots that were located in the 
four recesses of the epiphysis. Therefore, the recesses appeared to be the epiphyseal sites with the lowest bone masses, even though they exhibited higher vBMD values than the central zone of the diaphysis. Among these sites, the posterior medial malleolus had the lowest bone mass. We identified sites with more bone between these four spots and towards the medullary cavity. Although these sites were linked horizontally, they primarily occurred as vertical reinforcements that connected the cortical diaphysis with the subchondral articular bone. Other zones that showed higher bone mass were the epiphyseal plate and the subchondral cortical bone.

The results revealed a distinct bone mass pattern that varied across the anatomical sites examined. In the presence of osteopenia and osteoporosis, the bone mass was ubiquitously decreased. However, the data suggest disproportionate bone losses in the diaphysis, metaphysis, and epiphysis. These losses may reflect the increased endo- and intracortical, as well as trabecular, resorption. Our qualitative observations were confirmed using Meng's tests and were only detected in the anatomical regions with a relatively thick and dense cortical bone, such as those usually observed in the distal tibia. Further comparative analyses in normal, osteopenic, and osteoporotic bones are needed to confirm this observation. Other skeletal sites may be analysed using the same approach demonstrated in this study when DXA reference data are available.

We expected to see relatively thick cortical bone at the diaphyseal sites and relatively thin cortical bone at the epiphyseal sites in the normal, osteopenic, and osteoporotic models. The Ct.Th measurements were interpreted with regard to the manual approach and the image resolution of the HR-pQCT data. However, it was not the subject of this study to provide a more advanced measurement method. The maximal values of both of these parameters (i.e. Ct.Th and vBMD) were found in the proximal site, and both values gradually decreased towards and within the epiphysis. Accordingly, our models showed that the bone mass had decreased in all sites, while the pattern of bone distribution remained unchanged.

We were not able to detect any increased or decreased periosteal apposition as shown by alterations in the line curves of the virtual bore probes in the region under study. This result supports the observation made by Szulc et al. that periosteal apposition does not increase after menopause to compensate for bone loss. However, we were not able to detect decreased periosteal apposition either [23]. When comparing the line curves of the virtual bore probes, the vBMD values in the cortex of osteoporotic subjects were lower compared to those in normal and osteopenic ones, and likely a result of increased cortical porosity as reported by Zebaze and Seeman [24]. However, direct measurement of the cortical porosity is not possible at the used resolution in this study. Cortical thickness was assessed via the line curves of the virtual bore probes and the manual measurements. We expected the cortical thickness to be reduced at all the three sites within the same categorized group and among the three categorized groups. Direct measurement of the cortical thickness was a feasible yet a difficult task in subjects and sites with thin cortical thickness at the resolution used.

The two strengths of this study were the analysis of the distal tibia as our study site and the demonstration of the bone mass distribution and bone loss pattern in the presence of normal aBMD, osteopenia, and osteoporosis. The key limitation of the study was its cross-sectional character, which limited our ability to draw conclusions concerning bone loss. Despite this limitation, novel methods for creating 3D averaged models altered by bone loss and the analysis techniques were demonstrated. Furthermore, the use of HRpQCT data made cortical thickness measurements at the epiphysis difficult due to limited resolution, particularly in osteoporotic conditions. In contrast to cortical thickness measurements, we consider the cortical vBMD values to be lowered by increased cortical porosity as reported by Zebaze et al. [25]. However, the direct measurement of the cortical porosity is not possible at the resolution used. The results would have been more valuable if compared with the data obtained with conventional microarchitectural indices. However, it was not the aim of this study to evaluate conventional microarchitectural indices.

The data may also be used to predict the quality of the bone, to calculate the fracture risk, or to optimize implant constructs (e.g. when subjecting the models to numerical simulations, data mining, or machine learning). This study also suggests the ability of combined evidence from DXA and HR-pQCT imaging to compute 3D models to increase the knowledge about normal, osteopenic, and osteoporotic conditions. The approach is likely to improve future analysis, particularly with regard to the underlying bone loss mechanisms, and to enhance the understanding of local differences associated with normal, osteopenic, and osteoporotic conditions.

In conclusion, we presented an approach for spatially modelling the distal tibia and characterized the trabecular and cortical bone under normal, osteopenic, and osteoporotic conditions. Extra information can be obtained from the specific 3D pattern of bone mass distribution of the distal tibia in normal, osteopenic, and osteoporotic conditions, from potential disproportionate bone losses in the diaphysis, metaphysis, and epiphysis, and from the new technical approach modelling the entire distal length of the tibia.

Acknowledgments We thank Dr. Julian Erggelet for proofreading help. This research project was supported by AOTrauma from the AO Foundation, Davos, Switzerland (Research Grant: Trauma-11-05B). 


\section{Compliance with Ethical Standards}

Conflict of Interest Lukas Kamer, Hansrudi Noser, Michael Blauth, Mark Lenz, Markus Windolf and Albrecht W. Popp declare that they have no conflict of interest.

Ethical Approval An ethical waiver was provided by the local ethical committee stating that this study did not require an ethical approval.

\section{References}

1. Parfitt AM (1996) Skeletal heterogeneity and the purposes of bone remodeling: implications for the understanding of osteoporosis. In: Marcus R, Feldman D, Kelsey J (eds) Osteoporosis. Academic Press, San Diego, pp 315-339

2. Riggs BL, Melton LJ 3rd, Robb RA, Camp JJ, Atkinson EJ, Peterson JM, Rouleau PA, McCollough CH, Bouxsein ML, Khosla S (2004) Population-based study of age and sex differences in bone volumetric density, size, geometry, and structure at different skeletal sites. J Bone Miner Res 19:1945-1954

3. Boutroy S, Bouxsein ML, Munoz F, Delmas PD (2005) In vivo assessment of trabecular bone microarchitecture by high-resolution peripheral quantitative computed tomography. J Clin Endocrinol Metab 90:6508-6515

4. Davis KA, Burghardt AJ, Link TM, Majumdar S (2007) The effects of geometric and threshold definitions on cortical bone metrics assessed by in vivo high-resolution peripheral quantitative computed tomography. Calcif Tissue Int 81:364-371 (Epub 2007 Oct 20)

5. Melton LJ 3rd, Riggs BL, Keaveny TM, Achenbach SJ, Hoffmann PF, Camp JJ, Rouleau PA, Bouxsein ML, Amin S, Atkinson EJ, Robb RA, Khosla S (2007) Structural determinants of vertebral fracture risk. J Bone Miner Res 22:1885-1992

6. Mueller TL, Stauber M, Kohler T, Eckstein F, Müller R, van Lenthe GH (2009) Non-invasive bone competence analysis by high-resolution pQCT: an in vitro reproducibility study on structural and mechanical properties at the human radius. Bone 44:364-371. doi:10.1016/j.bone.2008.10.045

7. WHO Study Group (1994) Assessment of fracture risk and its application to screening for postmenopausal osteoporosis. Report of a WHO Study Group. World Health Organ Tech Rep Ser 843:1-12

8. Calori GM, Tagliabue L, Mazza E, de Bellis U, Pierannunzii L, Marelli BM, Colombo M, Albisetti W (2010) Tibial pilon fractures: which method of treatment? Injury 41:1183-1190

9. Ruetsche AG, Kneubuehl R, Birkhaeuser MH, Lippuner K (2005) Cortical and trabecular bone mineral density in transsexuals after long-term cross-sex hormonal treatment: a cross-sectional study. Osteoporos Int 16:791-798

10. Popp AW, Windolf M, Senn C, Tami A, Richards RG, Brianza S, Schiuma D (2012) Prediction of bone strength at the distal tibia by HR-pQCT and DXA. Bone. doi:10.1016/j.bone.2011.10.033
11. Kamer L, Noser H, Popp AW, Lenz M, Blauth M (2015) Computational anatomy of the proximal humerus: an ex vivo HRpQCT study. J Orthop Transl 4:46-56. doi:10.1016/j.jot.2015.09. 006

12. Biver E, Durosier C, Chevalley T, Herrmann FR, Ferrari S, Rizzoli R (2015) Prior ankle fractures in postmenopausal women are associated with low areal bone mineral density and bone microstructure alterations. Osteoporos Int 26:2147-2155. doi:10. 1007/s00198-015-3119-9

13. Popp AW, Senn C, Franta O, Krieg MA, Perrelet R, Lippuner K (2009) Tibial or hip BMD predict clinical fracture risk equally well: results from a prospective study in 700 elderly Swiss women. Osteoporos Int 20:1393-1399. doi:10.1007/s00198-0080808-7

14. Casez JP, Troendle A, Lippuner K, Jaeger P (1994) Bone mineral density at distal tibia using dual-energy X-ray absorptiometry in normal women and in patients with vertebral osteoporosis or primary hyperparathyroidism. J Bone Miner Res 9:1851-1857

15. Bookstein FL (1991/1997) Morphometric tools for landmark data. Cambridge University Press, Cambridge

16. Noser H, Hammer B, Kamer L (2010) A method for assessing 3D shape variations of fuzzy regions and its application on human bony orbits. J Digit Imaging 23:422-429. doi:10.1007/s10278010-9287-4

17. Kamer L, Noser H, Schramm A, Hammer B (2010) Orbital form analysis: problems with design and positioning of precontoured orbital implants: a serial study using post-processed clinical CT data in unaffected orbits. Int J Oral Maxillofac Surg 39:666-672. doi:10.1016/j.ijom.2010.03.005

18. Meng XL, Rosenthal R, Rubin DB (1992) Comparing correlated correlation coefficients. Psychol Bull 111:172-175

19. Dryden IL, Mardia KV (1998) Statistical shape analysis. Wiley series in probability and statistics. Wiley, New York

20. Styner MA, Rajamani KT, Nolte LP, Zsemlye G, Szekely G, Taylor CJ, Davies RH (2003) Evaluation of 3D correspondence methods for model building. Inf Process Med Imaging 18:63-75

21. Zollikofer CPE, Ponce de León M (2005) Virtual reconstruction, a primer in computer-assisted paleontology and biomedicine. Wiley, New York

22. Wähnert D, Hoffmeier KL, Lehmann G, Fröber R, Hofmann GO, Mückley T (2009) Temperature influence on DXA measurements: bone mineral density acquisition in frozen and thawed human femora. BMC Musculoskelet Disord 10:25. doi:10.1186/ 1471-2474-10-25

23. Szulc P, Seeman E, Duboeuf F, Sornay-Rendu E, Delmas PD (2006) Bone fragility: failure of periosteal apposition to compensate for increased endocortical resorption in postmenopausal women. J Bone Miner Res 21:1856-1863

24. Zebaze R, Seeman E (2015) Cortical bone: a challenging geography. J Bone Miner Res 30:24-29. doi:10.1002/jbmr.2419

25. Zebaze RM, Ghasem-Zadeh A, Bohte A, Iuliano-Burns S, Mirams M, Price RI, Mackie EJ, Seeman E (2010) Intracortical remodelling and porosity in the distal radius and post-mortem femurs of women: a cross-sectional study. Lancet 15:1729-1736. doi:10.1016/S0140-6736(10)60320-0 